\title{
Survival Analysis of Re-resection Versus Radiofrequency Ablation for Intrahepatic Recurrence After Hepatectomy for Hepatocellular Carcinoma
}

\author{
Albert C. Y. Chan • Ronnie T. P. Poon - Tan To Cheung • \\ Kenneth S. H. Chok $\cdot$ See Ching Chan · Sheung Tat Fan • \\ Chung Mau Lo
}

Published online: 27 October 2011

(c) The Author(s) 2011. This article is published with open access at Springerlink.com

\begin{abstract}
Background Tumor recurrence after resection of hepatocellular carcinoma is a common phenomenon. Re-resection and radiofrequency ablation (RFA) are good options for treating recurrent HCC. This study compared the efficacy of these two modalities in the treatment of intrahepatic HCC recurrence after hepatectomy.

Methods From January 2001 to December 2008, a total of 179 patients developed intrahepatic HCC recurrence after hepatectomy. To treat the recurrence, 29 patients underwent re-resection and 45 patients had RFA. Patient characteristics, clinicopathologic data, and survival outcomes were reviewed.

Results Child-Pugh status, time to develop first recurrence (12.2 vs. 8.7 months), and recurrent tumor size (2.1 vs. $2.1 \mathrm{~cm}$ ) were comparable for the two groups. Time to develop a second intrahepatic recurrence after re-resection and RFA was 5.9 and 4.0 months respectively. The 1-, 3-, and 5-year disease-free survival rates were $41.4 \%, 24.2 \%$, and $24.2 \%$ after re-resection and $32.2 \%, 12.4 \%$, and $9.3 \%$ after RFA $(p=0.14)$. The 1-, 3-, and 5-year overall survival rates were $89.7 \%, 56.5 \%$, and $35.2 \%$ after re-resection and $83.7 \%, 43.1 \%$, and $29.1 \%$ after RFA $(p=0.48)$. For the second recurrence, $33.3 \%$ of patients underwent a second round of RFA and $10.0 \%$ underwent a third resection.
\end{abstract}

A. C. Y. Chan $(\bowtie) \cdot$ R. T. P. Poon - T. T. Cheung

K. S. H. Chok - S. C. Chan - S. T. Fan - C. M. Lo Department of Surgery, The University of Hong Kong, Queen Mary Hospital, 102 Pok Fu Lam Road, Hong Kong L454, China e-mail: acchan@hku.hk

R. T. P. Poon - S. C. Chan - S. T. Fan - C. M. Lo State Key Laboratory for Liver Research,

The University of Hong Kong, Hong Kong, China
Conclusions The two treatment modalities attained similar survival benefits in the management of recurrent HCC after hepatectomy. The high repeatability of RFA and that it can be delivered percutaneously render it a preferred treatment option for selected patients.

\section{Introduction}

Hepatic resection remains an important curative treatment for hepatocellular carcinoma (HCC). Nonetheless, postresection tumor recurrence is common with a 5-year recurrence rate of $>50 \%[1,2]$. The treatment algorithm for recurrent HCC varies from center to center, and there is not yet a consensus on the most appropriate treatment for postresection tumor recurrence. Nonsurgical treatment such as transarterial oily chemoembolization is, at best, a palliative treatment after which long-term survival is seldom observed [3, 4]. Salvage transplantation probably produces the best survival results as it removes both the tumor and the cirrhotic liver [5, 6]. However, a shortage of liver grafts, stringent patient selection criteria, and a normal liver biochemical profile that constitutes a low Model for End-stage Liver Disease score often relegate patients with recurrent HCC to the least priority to receive a graft before the disease progresses. Re-resection would be an ideal alternative, which was shown to be an effective approach to prolong survival [7], but its feasibility is limited by the small liver remnant and inadequate liver function reserve. Another treatment option is radiofrequency ablation (RFA), which that is applicable even to patients with borderline liver function and can be delivered via the percutaneous as well as the open approach. The aim of this study was to determine if RFA or re-resection is the preferred treatment modality for the management of intrahepatic recurrence after hepatectomy for HCC. 


\section{Patients and methods}

From January 2001 to December 2008, 669 patients underwent hepatic resection for HCC at the Department of Surgery in Queen Mary Hospital, The University of Hong Kong. At the time of the data analysis, 257 patients remained free of recurrence, 179 patients had developed intrahepatic recurrence, 115 patients haddeveloped extrahepatic recurrence, and 118 patients had developed both. Among the 179 patients with intrahepatic recurrence, 74 patients underwent either re-resection or RFA as the secondary treatment, and they formed the focus of interest in this study. Prospectively collected data on patient characteristics, clinicopathologic features, and survival outcomes were reviewed.

\section{Diagnostic criteria for recurrent $\mathrm{HCC}$}

The follow-up protocol for patients after hepatectomy for primary HCC in our center consisted of computed tomography (CT) or magnetic resonance imaging (MRI) of the liver 1 month after hepatic resection to confirm macroscopic tumor clearance. Thereafter, surveillance for tumor recurrence was conducted using CT or MRI scans of the liver every 3 months with serial measurements of serum $\alpha$-fetoprotein (AFP) and liver biochemistry. Intrahepatic recurrence is defined as a new lesion with arterial enhancement and portal venous washout on contrastenhanced CT or MRI scans with or without an elevated serum AFP level (>200 ng/ml).

\section{Selection criteria for re-resection or RFA} as a secondary treatment

Only patients with Child-Pugh class A cirrhosis and selected patients with Child-Pugh class B cirrhosis were considered for re-resection or RFA. The usual indication for re-resection was a solitary or oligonodular tumor irrespective of tumor size within a monosegment of liver in the presence of a sufficient future liver remnant. It was generally avoided in the presence of (1) gross ascites, and/or (2) an indocyanine green retention rate of $>15 \%$ at $15 \mathrm{~min}$, and/or (3) a platelet count $<100 \times 10^{9} / \mathrm{L}$, signifying severe portal hypertension. RFA was selected when the recurrent tumor was (1) $<6 \mathrm{~cm}$ or there were three or fewer tumor nodules, and/or (2) in a deep-seated intraparenchymal location where anatomic re-resection would remove more than one segment of liver, leading to insufficient remnant liver volume. Needle biopsy for histologic confirmation was performed before application of RFA, and the tract was subsequently ablated on withdrawal of the RFA probe. The percutaneous approach was usually considered first $(n=22)$ unless the tumor was situated near the gallbladder or the bowel loops or when there was difficulty localizing the tumor with transabdominal ultrasonography. The open or laparoscopic approach was selected otherwise $(n=23)$.

Statistical analysis

Continuous variables were expressed in medians (interquartile range) and compared using the Mann-Whitney $U$-test. Categoric variables were compared with the $\chi^{2}$ test or Fisher's exact test when appropriate. Overall survival and disease-free survival of the two study groups were estimated by the Kaplan-Meier method. Comparison of survival between groups was performed with the log-rank test. Disease-free survival after treatment of intrahepatic recurrence was defined as the period from the date of treatment of recurrent $\mathrm{HCC}$ to the date of the second tumor recurrence or death. Overall survival after hepatectomy was defined as the period from the date of hepatectomy for the primary HCC to the date of death related to any cause. Overall survival after treatment of intrahepatic recurrence was defined as the period from the date of treatment for the first recurrence to the date of death related to any cause. A $p$ value $\leq 0.05$ was considered to be significant. Statistical analysis was performed using the SPSS 16.0 computer software program.

\section{Results}

The median follow-up duration was 44.9 months (8.3-112.0 months). Table 1 shows the patients' characteristics and liver function profiles at the time of the intrahepatic recurrence. Patients in the RFA group were older than those in the re-resection group. The two groups had a similar incidence of co-morbid illness and similar viral hepatitis carrier rates. All patients in the re-resection group had Child-Pugh class A cirrhosis, whereas $88.9 \%$ of patients in the RFA group were of Child-Pugh class A and $11.1 \%$ were of Child-Pugh class B $(p=0.17)$. Pretreatment liver biochemistry and renal function at the time of recurrence were comparable for the two groups except that patients in the RFA group had a higher serum bilirubin level and a lower serum albumin level. With regard to the pathology of the primary HCC (Table 2), the two groups shared similar features in terms of tumor nodularity, degree of tumor cell differentiation, and distribution of tumor staging [American Joint Committee on Cancer (AJCC) 6th edition). The RFA group, however, had a larger primary tumor size than the re-resection group $(5.5$ vs. $3.5 \mathrm{~cm}$, $p=0.01$ ), suggesting that tumors in the former group might be more aggressive. Time to develop first recurrence was longer in the re-resection group, although the difference was not statistically significant (12.2 vs. 8.7 months, 
Table 1 Patient characteristics and liver function profile at the time of recurrence after hepatectomy

\begin{tabular}{|c|c|c|c|}
\hline Characteristic & $\begin{array}{l}\text { Re-resection } \\
(n=29)\end{array}$ & $\begin{array}{l}\text { RFA } \\
(n=45)\end{array}$ & $p$ \\
\hline Age (years) & $52(38-79)$ & $59(36-80)$ & 0.03 \\
\hline Co-morbid illness & $11(37.9 \%)$ & $23(51.1 \%)$ & 0.27 \\
\hline Carrier of hepatitis B virus & $26(89.7 \%)$ & $40(88.9 \%)$ & 1.00 \\
\hline Carrier of hepatitis $\mathrm{C}$ virus & $1(3.6 \%)$ & $3(7.1 \%)$ & 0.39 \\
\hline Child-Pugh class A & $29(100 \%)$ & $40(88.9 \%)$ & 0.17 \\
\hline Child-Pugh class B & 0 & $5(11.1 \%)$ & \\
\hline Serum bilirubin $(\mu \mathrm{mol} / \mathrm{L})$ & $11(6-35)$ & $14(5-61)$ & 0.02 \\
\hline $\begin{array}{l}\text { Serum alanine } \\
\text { transaminase }(\mu / \mathrm{L})\end{array}$ & $51(17-99)$ & $47.5(12-379)$ & 0.83 \\
\hline $\begin{array}{l}\text { Serum aspartate } \\
\text { transaminase }(\mu / \mathrm{L})\end{array}$ & $48(19-77)$ & $43(20-177)$ & 0.76 \\
\hline Serum albumin $(\mathrm{g} / \mathrm{dl})$ & $41(28-48)$ & $39(25-49)$ & 0.03 \\
\hline Platelet count $\left(\times 10^{9} / \mathrm{L}\right)$ & $136(79-270)$ & $142(46-291)$ & 0.83 \\
\hline Serum urea $(\mathrm{mmol} / \mathrm{L})$ & $4.9(2.6-10.5)$ & $5.4(2.6-20.6)$ & 0.23 \\
\hline Serum creatinine $(\mu \mathrm{mol} / \mathrm{L})$ & $82(44-137)$ & $89(49-185)$ & 0.23 \\
\hline \multicolumn{4}{|c|}{ Nontumorous liver parenchyma } \\
\hline Normal & $4(13.8 \%)$ & $5(11.1 \%)$ & 0.17 \\
\hline $\begin{array}{l}\text { Chronic hepatitis/ } \\
\text { cirrhosis }\end{array}$ & $25(86.2 \%)$ & $40(88.9 \%)$ & \\
\hline
\end{tabular}

$R F A$ radiofrequency ablation

Table 2 Pathologic features of primary HCC

\begin{tabular}{lccc}
\hline Feature & $\begin{array}{l}\text { Re-resection } \\
(n=29)\end{array}$ & RFA $(n=45)$ & $p$ \\
\hline Primary tumor size $(\mathrm{cm})$ & $3.5(1.0-14.5)$ & $5.5(1.5-22.0)$ & 0.01 \\
$\begin{array}{l}\text { No. of tumor nodules } \\
\text { Tumor cell }\end{array}$ & $1(1-3)$ & $1(1-$ multiple $)$ & 0.11 \\
$\quad$ differentiation ${ }^{\mathrm{a}}$ & & & 0.86 \\
$\quad$ Well differentiation & $4(13.8 \%)$ & $8(17.8 \%)$ & \\
$\quad$ Moderate & $21(72.4 \%)$ & $29(64.4 \%)$ & \\
$\quad$ differentiation & & & \\
Poor differentiation & $4(13.8 \%)$ & $6(13.3 \%)$ & \\
Serum $\alpha$-fetoprotein & $64(2-167,138)$ & $90(1-197,122)$ & 0.85 \\
$\quad$ ng/ml) & & & 0.12 \\
AJCC stage & & $18(40.0 \%)$ & \\
I & $12(41.4 \%)$ & $19(42.2 \%)$ & \\
II & $15(51.7 \%)$ & $8(17.8 \%)$ & \\
III & $2(6.9 \%)$ &
\end{tabular}

HCC hepatocellular carcinoma, AJCC American Joint Committee on Cancer

a Two patients in the RFA group had a sampling error in the tumor biopsy specimen

$p=0.84)$. Pathological characteristics of recurrent tumors (Table 3) were similar in the two groups in terms of tumor size $(p=0.73)$, tumor nodularity $(p=0.48)$, and serum AFP level $(p=0.85)$. Table 4 showed the postoperative
Table 3 Tumor characteristics of intrahepatic HCC recurrence

\begin{tabular}{llll}
\hline Characteristic & $\begin{array}{l}\text { Re-resection } \\
(n=29)\end{array}$ & $\begin{array}{l}\text { RFA } \\
(n=45)\end{array}$ & $p$ \\
\hline $\begin{array}{l}\text { Time to recurrence } \\
\text { (months) }\end{array}$ & $12.2(1.8-84.3)$ & $8.7(1.0-88.5)$ & 0.84 \\
$\begin{array}{l}\text { Tumor size (cm) } \\
\begin{array}{l}\text { Solitary tumor } \\
\text { recurrence }\end{array}\end{array}$ & $2.1(0.8-5.5)$ & $2.2(0.8-6.0)$ & 0.73 \\
$\begin{array}{l}\text { Serum } \alpha \text {-fetoprotein } \\
(\text { ng/ml })\end{array}$ & $64(2-167,138)$ & $90(1-197,122)$ & 0.85 \\
\hline
\end{tabular}

Table 4 Postoperative outcomes after treatment of recurrent HCC

\begin{tabular}{llll}
\hline Outcome & $\begin{array}{l}\text { Re-resection } \\
(n=29)\end{array}$ & $\begin{array}{l}\text { Open RFA } \\
(n=23)\end{array}$ & $p$ \\
\hline Postoperative morbidities (no.) & $7(24.1 \%)$ & $2(8.7 \%)$ & 0.27 \\
$\quad$ Pleural effusion & 5 & 1 & 0.33 \\
Pneumonia & 1 & 1 & 1.00 \\
Wound infection & 3 & 0 & 0.32 \\
Intraabdominal hemorrhage & 1 & 0 & 1.0 \\
Septicemia & 1 & 0 & 1.0 \\
Hospital mortality (no.) & 0 & 1 & 0.91 \\
Dindo-Clavien classification $[20]$ & & & \\
II & 8 & 2 & \\
IIIa & 1 & 0 & \\
IIIb & 1 & 0 & \\
IVa & 1 & 0 & \\
V & 0 & 1 & \\
\hline
\end{tabular}

outcomes of patients undergoing re-resection and RFA via the open or laparoscopic approach $(n=23)$. Seven patients developed a total of 11 procedure-related morbidities in the re-resection group, with most of them Dindo-Clavien grade II. One patient in the RFA group developed aggressive lymphoproliferative disease after open RFA and succumbed during the same hospital admission. The incidences of procedure-related morbidity $(p=0.27)$ and hospital mortality $(p=0.91)$ were not statistically different for the two groups.

Table 5 illustrates the patterns of the second recurrence in the two groups after treatment of the first intrahepatic recurrence and the subsequent management. At the time of analysis, $27.6 \%$ of the patients in the re-resection group and $15.6 \%$ of the patients in the RFA group remained free of recurrence. The time to develop a second recurrence was 5.9 months in the re-resection group and 4.0 months in the RFA group $(p=0.30)$. By excluding tumors with a positive re-resection margin $(n=3,10.3 \%)$ and tumors that were incompletely ablated $(n=14,31.1 \%)$ during 
Table 5 Recurrence pattern and subsequent management after treatment of intrahepatic recurrence

\begin{tabular}{lcll}
\hline Recurrence & $\begin{array}{l}\text { Re-resection } \\
(n=29)\end{array}$ & $\begin{array}{l}\text { RFA } \\
(n=45)\end{array}$ & $p$ \\
\hline Recurrence pattern & & & 0.23 \\
$\quad$ No recurrence & $8(27.6 \%)$ & $7(15.6 \%)$ & \\
Intrahepatic only & $13(44.8 \%)$ & $19(42.2 \%)$ & \\
Extrahepatic only & $2(6.9 \%)$ & $1(2.2 \%)$ & \\
Intrahepatic and extrahepatic & $6(20.7 \%)$ & $18(40.0 \%)$ & \\
Treatment of second & & & 0.66 \\
intrahepatic recurrence & & & \\
Alcohol injection & $1(7.7 \%)$ & $0(0 \%)$ & \\
Transarterial oily & $5(38.5 \%)$ & $4(21.1 \%)$ & \\
$\quad$ chemoembolization & $1(7.7 \%)$ & $3(15.8 \%)$ & \\
Re-resection & 0 & $1(5.3 \%)$ & \\
Liver transplantation & 0 & $1(5.3 \%)$ & \\
Systemic chemotherapy & $3(23.1 \%)$ & $5(26.3 \%)$ & \\
RFA & $3(23.1 \%)$ & $5(26.3 \%)$ & \\
Conservative & & & \\
\hline
\end{tabular}

treatment for the first recurrence, the times to develop a second recurrence in the re-resection group and the RFA group was extended to 6.3 months and 9.5 months, respectively $(p=0.25)$. The $1-, 3-$, and 5-year overall survival rates from the time of hepatectomy for primary $\mathrm{HCC}$ in the re-resection group were $89.7 \%, 82.3 \%$, and $55.7 \%$, respectively. The RFA group had corresponding rates of $95.6 \%, 68.2 \%$, and $44.5 \%$ (Fig. 1). The 1-, 3-, and 5 -year overall survival rates after treatment of the first intrahepatic recurrence in the re-resection group were $89.7 \%, 56.5 \%$, and $35.2 \%$, respectively. The RFA group had corresponding rates of $83.7 \%, 43.1 \%$, and $29.1 \%$ (Fig. 2). The 1-, 3-, and 5-year disease-free survival rates after treatment of the first intrahepatic recurrence in the re-resection group were $41.4 \%, 24.2 \%$, and $24.2 \%$, respectively. The corresponding rates in the RFA group were $32.2 \%, 12.4 \%$, and $9.3 \%$ (Fig. 3).

Univariate analysis of nine clinical parameters (Table 6) showed that time to the first intrahepatic recurrence after hepatectomy, time to the second recurrence after treatment of the first intrahepatic recurrence, and recurrence in more than one organ after treatment of the first intrahepatic recurrence were significant clinical factors that adversely affected overall survival after hepatectomy for primary HCC. In the multivariate analysis, only recurrence in more than one organ after treatment of the first intrahepatic recurrence remained an independent unfavorable prognostic factor for overall survival $(p<0.001$, hazard ratio 4.424, confidence interval 2.313-8.462).

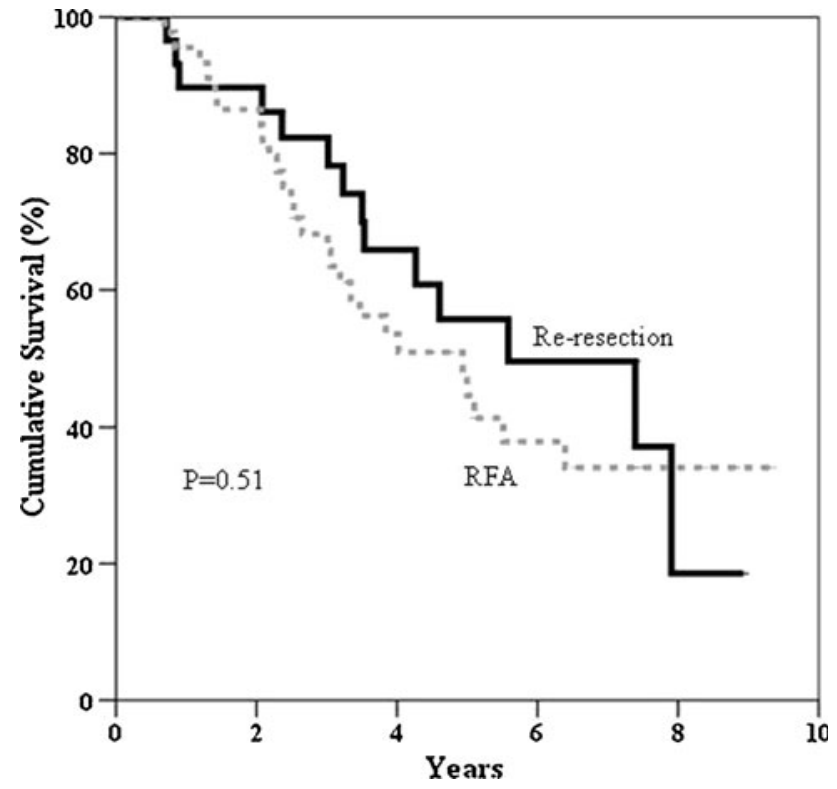

Fig. 1 Overall survival after hepatectomy for primary hepatocellular carcinoma. $R F A$ radiofrequency ablation

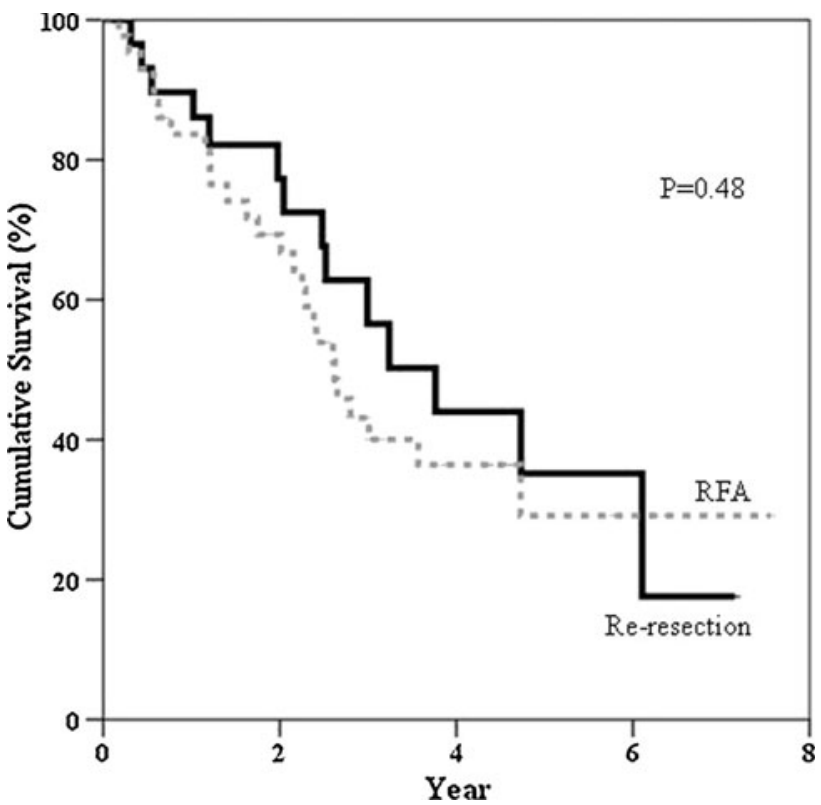

Fig. 2 Overall survival after treatment of intrahepatic recurrence

\section{Discussion}

With the refinement in preoperative liver function assessment [8] and surgical techniques for hepatectomy over the past decade leading to decreased postoperative morbidity and mortality $[9,10]$, more patients with cirrhotic livers are now amenable to major hepatectomy. Nonetheless, postresection tumor recurrence is common, with a 5-year recurrence rate $>50 \%[1,2,11,12]$. In all, $80 \%$ of the 


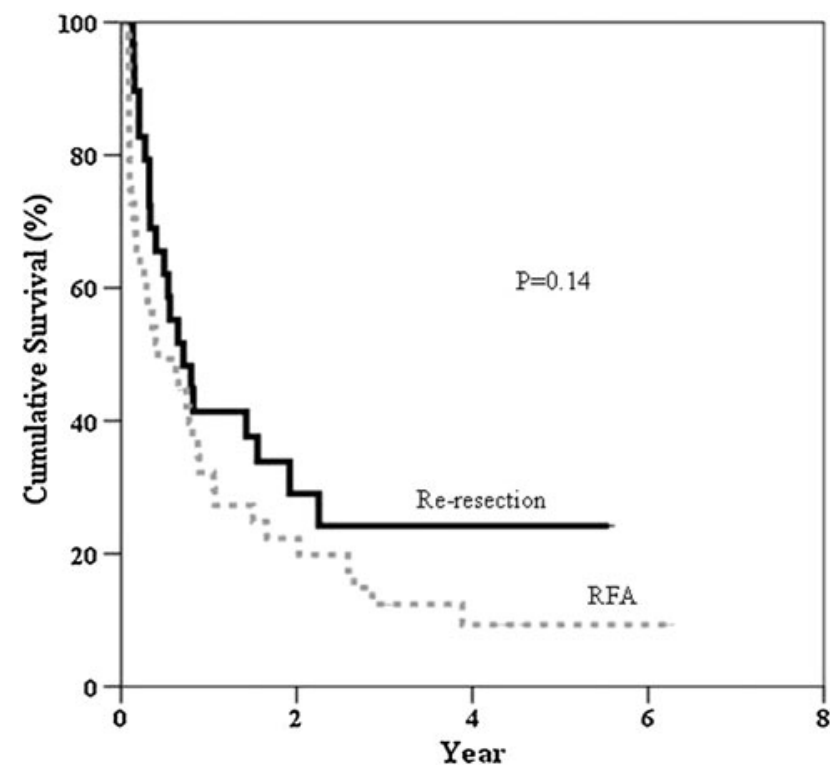

Fig. 3 Disease-free survival after treatment of intrahepatic recurrence

recurrences develop within the liver remnant [13], so it is important for surgeons to select the most appropriate treatment for patients with recurrent HCC. Cirrhosis is a known risk factor for intrahepatic recurrence [1, 13].

Treatment of intrahepatic recurrence poses several technical challenges, including a small liver remnant, inadequate liver function reserve, significant adhesion from a previous operation, and proximity of the tumor to major vascular or biliary structures. All of these conditions are relative contraindications to re-resection. The Kyoto group demonstrated a significant improvement in overall survival after active treatment of intrahepatic recurrence by either re-hepatectomy or RFA [14]. However, their study did not explore the most appropriate choice of surgical treatment for intrahepatic recurrence.

Our study showed that RFA could achieve long-term survival outcomes similar to those seen with re-resection in patients with recurrent HCC. After excluding confounding factors (positive resection margin, incomplete ablation) for further intrahepatic recurrence, the survival benefit of RFA remained unchanged. There are several reasons why RFA should be the preferred treatment option for intrahepatic recurrence. First, RFA can be delivered percutaneously, thereby avoiding a second operation. Second, RFA is applicable even to tumors proximal to major intrahepatic bile ducts. The practice of bile duct cooling (i.e., instillation of cold normal saline into the common bile duct via the cystic duct stump [15] or nasobiliary drain [16, 17]) can protect the bile duct from damage by the heat-sink effect of RFA. In our experience, about $3.5 \%$ of the patients undergoing RFA required bile duct cooling, and no biliary complications were observed in these patients [15]. Third,
Table 6 Univariate analysis identifying prognostic factors for overall survival after hepatectomy

\begin{tabular}{|c|c|c|}
\hline Clinical parameter & $\begin{array}{l}\text { Median survival } \\
\text { (months) }\end{array}$ & $p$ \\
\hline Age (years) & & 0.18 \\
\hline$\leq 55(n=38)$ & 51.20 & \\
\hline$>55(n=36)$ & 76.70 & \\
\hline Tumor size $(\mathrm{cm})$ & & 0.26 \\
\hline$\leq 5.0(n=46)$ & 66.97 & \\
\hline$>5.0(n=28)$ & 59.22 & \\
\hline Viral hepatitis positivity & & 0.56 \\
\hline Hepatitis B $(n=66)$ & 55.24 & \\
\hline Hepatitis C $(n=4)$ & 76.70 & \\
\hline Child-Pugh classification & & 0.78 \\
\hline $\mathrm{A}(n=70)$ & 59.97 & \\
\hline $\mathrm{B}(n=4)$ & 16.92 & \\
\hline Re-resection & & 0.19 \\
\hline Yes $(n=33)$ & 88.70 & \\
\hline No $(n=41)$ & 46.07 & \\
\hline $\begin{array}{l}\text { Time to first intrahepatic } \\
\text { recurrence }\end{array}$ & & $<0.001$ \\
\hline$\leq 12$ months $(n=38)$ & 6.67 & \\
\hline$>12$ months $(n=36)$ & 36.12 & \\
\hline Time to second tumor recurrence & & $<0.001$ \\
\hline$\leq 12$ months $(n=21)$ & 5.29 & \\
\hline$>12$ months $(n=19)$ & 30.23 & \\
\hline Single-site recurrence ${ }^{\mathrm{a}}$ & & $<0.001$ \\
\hline Yes $(n=48)$ & $>112.03$ & \\
\hline No $(n=26)$ & 36.64 & \\
\hline Intrahepatic recurrence & & 0.29 \\
\hline Solitary $(n=50)$ & 61.2 & \\
\hline Multiple $(n=24)$ & 46.1 & \\
\hline
\end{tabular}

\footnotetext{
${ }^{a}$ Denotes recurrence in one organ after treatment of a first intrahepatic recurrence
}

conservation of nontumorous liver parenchyma and negligible blood loss associated with RFA minimize the degree of surgical insult to the small and cirrhotic liver remnant. Fourth, repeatability is a major advantage of RFA. Our study showed that $26.3 \%$ of patients in the RFA group and $23.1 \%$ of patients in the re-resection group underwent RFA for their second intrahepatic recurrence, whereas less than a fifth of the patients in the RFA group and re-resection group were amenable to repeated resection for second intrahepatic recurrence. These four factors contribute to the safety and feasibility of RFA in the management of intrahepatic recurrence after hepatectomy.

Despite the fact that the chance of survival was improved by further surgical treatment, early first intrahepatic recurrence after hepatectomy, early second intrahepatic recurrence after either RFA or re-resection, and second recurrence in more than one organ remained poor 
prognostic factors for overall survival in patients with recurrent HCC after hepatectomy. Further research should therefore focus on adjuvant treatment after resection of a primary HCC. In fact, our recent study demonstrated the survival benefit of postresection antiviral therapy in antiviral-naive patients with early staged tumors [18]. Alternatively, results from the STORM trial [19] might identify a new avenue in the area of adjuvant treatment for HCC in the future.

Our study design was not without pitfalls: a small sample size, nonrandomization regarding choices of treatment, and selection bias. However, a randomized controlled trial might not be practical as fewer than $50 \%$ of the patients with a first intrahepatic recurrence were eligible for further surgical treatment in this study.

\section{Conclusions}

Radiofrequency ablation achieved long-term survival outcome similar to those seen with re-resection in the treatment of intrahepatic recurrence after hepatectomy. Secondary treatment of recurrent HCC after hepatectomy remains a challenging issue for hepatobiliary surgeons. However, a persevering attitude is mandatory in the longterm management of $\mathrm{HCC}$ to improve the chance of treatment for recurrent disease. The fact that RFA can be delivered percutaneously and its high repeatability render it a preferred treatment option in selected patients with HCC recurrence.

Open Access This article is distributed under the terms of the Creative Commons Attribution Noncommercial License which permits any noncommercial use, distribution, and reproduction in any medium, provided the original author(s) and source are credited.

\section{References}

1. Grazi GL, Cescon M, Ravaioli M et al (2003) Liver resection for hepatocellular carcinoma in cirrhotics and noncirrhotics: evaluation of clinicopathologic features and comparison of risk factors for long-term survival and tumour recurrence in a single centre. Aliment Pharmacol Ther 17(Suppl 2):119-129

2. Inoue K, Takayama T, Higaki T et al (2004) Clinical significance of early hepatocellular carcinoma. Liver Transpl 10(Suppl 1): S16-S19

3. Choi JW, Park JY, Ahn SH et al (2009) Efficacy and safety of transarterial chemoembolization in recurrent hepatocellular carcinoma after curative surgical resection. Am J Clin Oncol 32:564-569

4. Shim JH, Kim KM, Lee YJ et al (2010) Complete necrosis after transarterial chemoembolization could predict prolonged survival in patients with recurrent intrahepatic hepatocellular carcinoma after curative resection. Ann Surg Oncol 17:869-877

5. Belghiti J, Cortes A, Abdalla EK et al (2003) Resection prior to liver transplantation for hepatocellular carcinoma. Ann Surg 238:885-892 discussion 892-893

6. Hwang S, Lee SG, Moon DB et al (2007) Salvage living donor liver transplantation after prior liver resection for hepatocellular carcinoma. Liver Transpl 13:741-746

7. Minagawa M, Makuuchi M, Takayama T et al (2003) Selection criteria for repeat hepatectomy in patients with recurrent hepatocellular carcinoma. Ann Surg 238:703-710

8. Fan ST (2010) Liver functional reserve estimation: state of the art and relevance for local treatments-the Eastern perspective. J Hepatobiliary Pancreat Sci 17:380-384

9. Fan ST (2004) Protection of the liver during partial hepatectomy. Hepatobiliary Pancreat Dis Int 3:490-494

10. Fan ST, Lo CM, Liu CL et al (1999) Hepatectomy for hepatocellular carcinoma: toward zero hospital deaths. Ann Surg 229: 322-330

11. Ercolani G, Grazi GL, Ravaioli M et al (2003) Liver resection for hepatocellular carcinoma on cirrhosis: univariate and multivariate analysis of risk factors for intrahepatic recurrence. Ann Surg 237:536-543

12. Poon RT, Fan ST, Lo CM et al (2000) Long-term prognosis after resection of hepatocellular carcinoma associated with hepatitis B-related cirrhosis. J Clin Oncol 18:1094-1101

13. Poon RT, Fan ST, Wong J (2000) Risk factors, prevention, and management of postoperative recurrence after resection of hepatocellular carcinoma. Ann Surg 232:10-24

14. Taura K, Ikai I, Hatano E et al (2006) Implication of frequent local ablation therapy for intrahepatic recurrence in prolonged survival of patients with hepatocellular carcinoma undergoing hepatic resection an analysis of 610 patients over 16 years old. Ann Surg 244:265-273

15. Lam VW, Ng KK, Chok KS et al (2008) Safety and efficacy of radiofrequency ablation for periductal hepatocellular carcinoma with intraductal cooling of the central bile duct. J Am Coll Surg 207:e1-e5

16. Ogawa T, Kawamoto H, Kobayashi Y et al (2010) Prevention of biliary complication in radiofrequency ablation for hepatocellular carcinoma: cooling effect by endoscopic nasobiliary drainage tube. Eur J Radiol 73:385-390

17. Nakata Y, Haji S, Ishikawa $H$ et al (2010) Two cases of hepatocellular carcinoma located adjacent to the Glisson's capsule treated by laparoscopic radiofrequency ablation with intraductal chilled saline perfusion through an endoscopic nasobiliary drainage tube. Surg Laparosc Endosc Percutan Tech 20: e189-e192

18. Chan AC, Chok KS, Yuen WK et al (2011) Impact of antiviral therapy on the survival of patients after major hepatectomy for hepatitis B virus-related hepatocellular carcinoma. Arch Surg 146:675-681

19. Printz C (2009) Clinical trials of note: sorafenib as adjuvant treatment in the prevention of disease recurrence in patients with hepatocellular carcinoma (HCC) (STORM). Cancer 115:4646

20. Dindo D, Demartines N, Clavien PA (2004) Classification of surgical complications: a new proposal with evaluation in a cohort of 6336 patients and results of a survey. Ann Surg 240:205-213 